\title{
Exploring the Video Productions of Egyptian Applied Arts Stu- dents
}

\author{
By Mustafa Mahmoud Yousry \\ Spring 2013 Issue of KINEMA
}

\section{EXPLORING THE VIDEO PRODUCTIONS OF EGYPTIAN APPLIED ARTS STUDENTS}

\begin{abstract}
This paper summarizes the key findings identified during a research project on the use of video for youth selfexpression and communication. The project was conducted within the department of Photography, Cinema and Television, Faculty of Applied Arts, Helwan University (Egypt), where this researcher works as a lecturer of media production. Over one academic year, and during a level-3 media production course, the researcher was involved in teaching 16 young Egyptian students from different background, how to produce short videos. In groups, the students planned and produced several short videos on a range of themes. Afterwards, selections from the students' productions were presented to a sample of 23 adolescents representing the students in other academic levels from the same department. The viewers watched the produced videos and their responses to each production were observed and analyzed. The viewers' comments were also directed to the young videomakers, and the reactions of those young creators were recorded and discussed as well.
\end{abstract}

\section{Introduction}

The importance of video and television in young people's life experiences is well established through research on media consumption (Buckingham, 2000; Fisherkeller, 2002). By contrast, there is very little documentation and research on young people as video producers and on youths' interpretations of other youths' video productions. In other words, several analyses of films and television programmes about young people exist, but very little attention has been paid to short films and videos made by young people themselves. However, research of this kind is now beginning to emerge in the fields of visual sociology and anthropology. Besides analyzing the films and videos themselves, researchers are gathering data about the contextual aspects of the production process (Niesyto, 2001). The researchers need to understand how young people incorporate cultural resources made accessible to them in everyday life and to examine the modes of expression they adopt in doing so. In this process, research needs to develop new methodological approaches that go beyond verbal and written methods of collecting and recording data (Niesyto, 2001).

In fact, audiovisual media increasingly offer young people opportunities to communicate their ideas and feelings using nonverbal and non-print forms. The impressive, emotional, and ambiguous nature of a great deal of media material expands the norms and repertoires of representation expected by society, which are largely oriented around rationality and effectiveness. The field of media education gives young students opportunities to express personal and group-oriented experiences in self-produced videos. Such videos typically represent different physical forms as well as more abstract forms of symbolization.

Young Egyptian media students within the department of Photography, Cinema and Television, Faculty of Applied Arts, Helwan University, use low-cost analog and/or digital video cameras, non-linear editing software and notebook computers to create short video films. These video films are essentially simple stories and are made to fulfill the requirements of their academic courses. Frankly speaking, these videos are usually made with really limited resources, which might explain in part, why they are simple and short in most cases. The students make their videos from stories based on their own cultures and backgrounds. Stories that have previously been shared only with family and friends. Indeed, their social classes in the Egyptian society play a crucial role here.

Making video films is not easy to do but it can be learned. Media courses within the Faculty of Applied Arts are typically run for a full academic year over almost an eight-month period. The first-term lectures usually focus on script construction and the techniques of video image capturing. During the second term, the students, in groups, produce their own video films. Each group or work team of students usually consists of a project manager or producer, a scriptwriter, a cameraman, a video editor, and a director. 
In the making of short video films, the students are not just shooting their own material, they are editing it too. They shoot and record perhaps for hours and then keep only those bits that tell their stories their own way. They feel they are creating their own media. Consequently, video making cannot be considered as just a course applied-arts students take; if they learn the skills of making short video films, then they can, quite literally, 'take the power back.' An individual relates himself in action to his society through the use of tools that he actively masters, or by which he is passively acted upon. To the degree that he masters his tools, he can invest the world with his meaning; to the degree that he is mastered by his tools, the shape of the tool determines his own self-image. Convivial tools are those which give each person who uses them the greatest opportunity to enrich the environment with the fruits of his or her vision (Illich, 2001). Until now, broadcasting, especially television, has been - as Illich put it - an 'un-convivial tool'. For television as we know it 'acts passively upon individuals'. What video filmmaking courses do is open up the possibility that students can turn the television experience around, become their 'active masters.'

To us at Helwan University, media courses in general, and teaching the making of short video films in particular, have the following considerable benefits:

- Fresh output from the students reflecting on the issues of their own communities.

- A new way for the University to connect with communities, not in a top-down corporate manner but through projects which depend upon actions within communities for its delivery and success.

- An original and sustainable contribution to enhance students' self-expression.

- Opening up new lines of talent in journalism, videography, editing, scriptwriting, directing and visual skills.

- Access for Egyptian young students to the latest multimedia technology which is vital to the future.

- An opportunity for lecturers and researchers to apply their knowledge on the ground, which also could provide hugely valuable examples for the spread of community use of media technology.

- Most of the students who participate in producing short video films believe that in telling stories, communities and individuals assert their identity and escape from the confines of dominant images generated by mass media. They believe that they raise community self-esteem.

\section{Research questions}

The present research project attempts to address the following questions:

1. In producing videos, do young Egyptian applied arts students disconnect themselves from the constraints of their immediate social norms and background repertoires?

2. Through video productions, are young Egyptian applied arts students able to establish new forms of contact with different youth backgrounds?

3. To what extent can we identify a trans-cultural, audiovisual, symbolic language in videotapes produced by groups of young Egyptian applied arts students from different backgrounds?

4. To what extent are the symbolic styles and interpretations influenced by education, gender, and class background, as well as by the characteristics of young Egyptian applied arts students' own media cultures and economic resources?

\section{Methodology}

Within qualitative youth and communication research, verbally based methods such as narrative interviews, group discussions, or written field notes are still predominant. Yet these methods may provide limited access to the emotional and symbolic aspects of young students' experiences and media-related modes of expression. The methodology that is applied in this project acknowledges the significance of audiovisual communication in the students' experiences of reality and offers a new perspective for youth research. In simple words, to learn about young students' views and perspectives, we should give them opportunities to express themselves through their own media productions, as well as share their creations with other students.

In this project, the researcher wanted to build on previous work about young people's uses of media for creative production (e.g., Buckingham and Sefton-Green 1994; and Buckingham et al., 1995). The concept was to give the chance to groups of Egyptian applied arts students between the ages of eighteen and twenty to 
produce, exchange, and interpret thematically oriented video productions. The groups consisted of students from a variety of social and economic backgrounds. At the beginning, the sixteen level-3 students were asked to deal in their videos with ideas and themes representing their own Egyptian society and culture. They were given full freedom to discuss any stories and/or issues that stemmed from that general outline. Over all, ten video films were produced by the students representing a mixture of topics, video styles, and social backgrounds.

During the course of this research work, several methods were used to record and analyze the sequence of events. During classes, participating observers focused on the production processes, communication among students, and conversations between them and the researcher. Afterwards, open questionnaires and group discussions were used and transcripted to gather individual impressions and interpretations of the presented video films (Lamb, 2003). Finally, the video films themselves were analyzed using a range of criteria developed from the students' own interpretations and focused largely on aspects of image, sound, and editing.

\section{Categories of the selected video films}

For the purposes of this research work, and with the help of some fellow lecturers, the students' finished video films were reviewed by the researcher and two distinct categories were made for presentation and examination. These two contrasting and differing categories of video films were screened in front of a sample group of 23 applied arts students from culturally and socially diverse backgrounds who are themselves videomakers.

In the video films of the first category there are clear sequences of establishing shots and close-ups, shot reverse shot patterns, and point-of-view shots. The images are accompanied most of the time by suitable sound tracks (e.g., classical music, fast drum-and-bass sound, etc.). After analyzing the personal and background data of the makers of the video films in this first category, it was found that the majority of them come from working-class backgrounds. Following the initial lectures provided by the lecturer/researcher at the beginning of the course, these groups of students spent some time casting around for ideas. Actually, they expressed some frustration with the researcher's notion of depending mainly on the visuals in their productions and to use limited or even no language or dialogue at all. They felt that dialogues would be needed to convey their "stories," and never considered the possibility of presenting their ideas in non-narrative forms. Broadly speaking, however, they were pleased with the final results; the researcher felt that these groups had developed an effective grasp of the "film language" he had set out to teach.

On the other hand, the video productions of the second category consist of a very different kind of video films. Although, these films focus on stories and themes related to the Egyptian society and culture, just as the videos of the first category, they are constructed in the shooting and editing-based styles of advertising and music videos. For example, strange and oblique shooting angles are obvious, no regard of the correct images sizes-order is being taken into account, lighting styles that contradict well-known traditions and techniques are frequently used, some images are reflected in mirrors, and the characters frequently look directly into the camera. These video films are accompanied by soundtracks that match the fast nature of their images and editing styles.

After analyzing the personal and background data of the video-makers in this second category, it was found that the majority of them are from a higher class (i.e., they enjoy economic and social advantages that the first groups of students do not have), and most of them are high achievers in terms of formal education. Both their video productions and their subsequent contributions to the interpretation discussions reflect the fact that they have an adequate academic orientation towards "art." Many of them reported that when they had heard the course assignment for the first time, they had immediately decided to construct videos with "unique" shooting and fast editing styles. At an early stage during the project, these groups of students designed and produced storyboards, which remained close to their final edited versions. Their evaluation of the course was very positive. Many of them commented on enjoying the "freedom" to create their own video films.

In terms of film language, there are several striking differences between these two categories of video films. The groups that were involved in producing the first category of videos set out to produce narrative with a beginning, a middle, and an end. In this respect, they achieved their goals. The characters have coherent motivations, and one event leads logically to the next. Their productions employ the most obvious "rules" or conventions of continuity editing, and there is never any doubt about where the viewer is positioned in 
time or space. By contrast, the second category's groups set out to produce non-narrative, fast editing-style video films. Several recurrent images are intertwined although there is no obvious logic about how they are juxtaposed or arranged. While there are "characters" here, we are left to infer a good deal about what motivates them. The pace of the editing relates most of the time to the pace of the musical soundtracks, but there is hardly obvious use of continuity editing or clear sequence of cause and effect.

This kind of video productions requires more intensive forms of interpretative work on the part of the viewer, and it consciously promotes a degree of uncertainty (Messaris, 1995; Buckingham, 1993). Interestingly, the first group of video films was interpreted by other students who watched them as promoting a message although in fact their makers did not claim this as their overt intention. By contrast, the second group of video films specifically aims for an aesthetic response rather than a singular "meaning." Perhaps, these differences could be traced to the social and cultural differences between their makers (i.e., the students' various social classes and backgrounds are partly to do with their "sub-cultural" experiences and identifications). The second category group of students clearly possess a form of cultural capital that is unavailable to the students of the first group category.

\section{Presenting the video films and audiences' role}

The "different backgrounds" characteristic of the participating students enabled the researcher to pay particular attention to how young people develop their sense of audience. The introduction to the course included no explicit discussion among the working groups about how other students might view their video productions. It was only on subsequent reflection that the issue of audience really became a concern - and this emerged in particular when the groups had the opportunity to watch and discuss some of the videos produced by other groups involved in the course. The question which was generated during that stage of the project was: would it make a difference to these young videomakers if they knew at the beginning that their work would be seen by other fellow students and not just by their lecturer(s)? In fact, and as the situation in some other creative disciplines, most of what students produce in media classrooms is actually made for an audience of one - namely, the lecturer. In principle, enabling the students to produce for a "real" (i.e., non lecturer) audience can encourage them to think through the choices they make in production and their possible consequences more broadly. Being confronted with audience responses can motivate the students to reflect more critically on the relationships between intentions and results. Yet there may be limits to the extent to which students will be able to take account of any audience, however "real" (Buckingham et al., 1995). And even in the present case of this research work, questions remain about how real the audience might be.

Twenty three students were accommodated in a large television studio to view the two categories of young students' video productions selected by the researcher for presentation and interpretation. These eighteen to twenty-year-old viewers were asked to describe the content or topic of each of the video films, to identify and justify what they liked and disliked about each production, to suggest what they might do differently as videomakers themselves, and to specify what they would say to their colleagues. The titles and credits of the video films were not revealed until after the screening and discussion. Generally, and understandably, the students had varied responses to the video films. They had diverse backgrounds and life circumstances, and each of them had a unique point of view and style of interacting. Thus, they did not always agree on what these video films were about, whether they liked them, or why. In addition, group dynamics played a role in how comfortable and competent participants felt about discussing different aspects of these films (Buckingham 1993, 1996), so some voices prevailed while others were less dominant.

Among what the researcher focused on during this phase of the project was the students' responses to the discussed ideas and themes within the examined videos. Overall, these young audiences identified common aspects in the video films that they associated with their own lives. Communicating "youth experience" was stressed as the participating students were told that they would be viewing media products created by other students expressing something about their lives - which may have "cued" the students to focus on precisely these elements in their discussions. However, these viewers' connections with the videomakers' experiences also prompted them to suggest potential changes in the video films that would conform to their particular emotional and aesthetic sensibilities. The comments of this sample of young viewers were directed afterwards to the students who created the screened video films, and the reactions of those young creators were recorded and discussed as well. 
Initially, the groups of videomakers who created the first category of video films were unwilling to accept the suggestion - represented strongly in some of the viewers' feedbacks - that their video films were conveying "messages". They maintained that these messages had not been intentional on their part. As such, audiences' responses helped them to become aware of a gap between their intentions and the final results; thus, real audiences can play a role in students' learning about their own media productions. By contrast, the groups of videomakers who created the second category of videos had consciously seen the preparation and production stages of their videos as personal, or as an attempt to express something they felt on an emotional level. In other words, these groups did not really care that much if the audiences totally misunderstood or misinterpreted their videos; they did them for themselves in the first place. However, some of them also mentioned that they had changed to more distanced stances at the editing stage and made their selections on the basis of which shots might have a visual impact on the audience.

Over all, the video films of the second category were received with more considerable enthusiasm by the employed sample of "young" viewers. The responses of those students after watching these videos were fairly diverse, but in general, these videos were rated very highly. Interestingly, much of the subsequent discussions focused on the "symbolism" of these video films, to an extent that even their makers themselves found rather interesting. Actually, both in the presentation of their own work and in their reflections on other students' videos, the groups of videomakers who created the second category of video films were able to show aesthetic appreciation that was not articulated by other groups of students. For example, they judged other groups' productions in terms of existing cultural movements, they searched for "symbolism" and philosophical themes, they used a somewhat "technical" terminology, and they were able to assume a rational distance both from the productions themselves and from their own responses to them (Bourdieu, 1984). Likewise, in relation to their own work, they were keen to present themselves as artists with "vision" and "imagination," claiming their video films to be "personal" statements that were "clear and focused" from the very beginning. In fact, there are some grounds for questioning these claims, since at least some of what they produced came down to accidents in the editing phase.

However, in the point of view of the course leader (i.e., the researcher), it was the groups of students who created the first category of video films who have in fact learned the most - at least in terms of what the researcher claimed he had been attempting to teach. They had developed an ability to use the conventions of continuity editing to tell a simple story, which had been one of the aims of the course, and while other groups had not set out to do this, it is certainly debatable whether the students of those other groups learned anything that they did not already know. Here again, we need to avoid the temptation to merely celebrate youthful "creativity" and pay closer attention to the kinds of learning that it might entail. On the other hand, it should be stated that some other scholars have different opinions to that of the researcher. Fisherkeller et al. (2000), for one, argue in favour of associative, exploratory approaches, which encourage nonlinear forms of symbolization rather than narrative.

For example, the procedure of first taking everyday images, instead of striving for an immediate orientation towards a constructed plot (i.e., using a storyboard or script), enables the producers to see everyday life in a new light by trying out unusual angles and different distances. Some students come to know video films as a means of art which does not have a firm meaning or content in advance. However, from Fisherkeller and his colleagues' point of view, this depends on the tutors or lecturers who can give sensitive and competent advice, who provide young students with aesthetic input on different levels and in different situations, and who make sure that there is a constructive working atmosphere.

Nevertheless, for all the students who participated in the project, the experience of watching other video productions definitely influenced how they thought about their own, or at least what they were prepared to say about this. There was a kind of "de-centering" here: thinking about their own interpretations of other people's productions encouraged them to think about how other people would think about theirs. In particular, it led them to consider the relationship between intentions and results, to recognize that some of their intentions were not clear or had changed as the work progressed, and that some of the outcomes did not correspond to their initial intentions and may even have led to them being misinterpreted. The mere fact that there was a real audience (and indeed that they themselves were a real audience for somebody else's productions) seems to have helped them evaluate their own work in a more thoughtful and critical way. 


\section{Reflections on the participated students' comments}

Someone once suggested that all teens have "a hard life." Thus, it appears that those young viewers and videomakers see the majority of their fellow teens confronting a complex of responsibilities and living with a good deal of stress and strain, like themselves. Hence, despite some of their critiques of the screened video films, most of the students might have been intent to find resonance with the video film producers, whom they presumed were struggling like themselves. These are similarities they might not seek in other kinds of media productions presented in television for example, even if those productions feature teens, since these young viewers assume such productions have been produced by adults about youths, rather than by youths (Tally and Kornblum, 1997). Some participating students suggested that adults cannot communicate with teens as effectively as youths themselves because, as one student explained, things are different for adolescents now, compared to when current adults were young. This student argued that adults cannot really understand what is going on for youths today, even though adults, such as his own father, claim understanding when they invoke their own younger experiences.

What is important about this explanation is the logic it presents: these young Egyptian students are arguing that other students who come from different Egyptian backgrounds, and who live within other communities and regions, are more effective at communicating with them, compared to the adults in their own social classes or even in their own homes. Yet their connections with the young videomakers as youths might also be explained by their experiences in a city of multiple cultures and regional histories. Cairo teens are far from homogeneous: they come from a range of different social and cultural backgrounds - a situation characteristic of most large urban areas. Indeed, most of these young people, or their parents or grandparents, had emigrated from other parts of Egypt in recent years. Given these contexts, perhaps these youths found points of connection in the video films created by "others": they appeared to recognize a "we" even while they acknowledged that the films were created by young people who are socially and culturally different.

Some of these young viewers identified features of the video films that they felt should have been different from their expectations. Some others looked to find elements in the videos that were different from their own experiences. For example, while most of the viewers recognized popular songs and understood the tone of the music used in the video films, some of them suggested that the videomakers should have included music and soundtracks from their own Egyptian culture in the produced video films. Thus, while these youths wanted to make connections with the video films producers as youths, some wanted the youth producers to express aspects particular to their cultures and backgrounds as well.

Indeed, what was valued highly in the video films were the expressions and features of teens "being themselves" or "doing whatever they wanted." This is one reason that the video films of the second selected category were chosen by the majority of viewers as the ones they liked in particular. Even though they did not completely understand some of these video films, they found them creative and attention-grabbing due to the camera work, and editing style. But, in particular, the young viewers admired how the second category videomakers seemed to be saying "this is who we are" strongly and clearly, and "who they are" was seen as self-expressively different and bold. Indeed, this aspect of the videos prompted one participant to suggest that the video films of the second category be presented to adults as an exemplary statement about the nature of youths, so that adults could better understand adolescent experiences.

\section{Conclusions and recommendations}

Rarely in Egypt, and perhaps worldwide as well, are adolescents from relatively different socio-economic classes brought together for engaging in audiovisual communications like the ones which took place during the research project. This might be the main and most important benefit gained from the project. The research conclusions and recommendations could be summarized as follows:

1. Over all, ten video films were created during the course of the project. These videos contained different topics and modes of narrative and stylistic expressions. The characteristics and styles of these video films are partly a reflection of the particular contexts in which they were produced and of the individuals or groups involved in producing them. Such styles and characteristics were developed from the young videomakers' knowledge and previous experiences of media genres. These styles and characteristics also reflect broader social and cultural differences. In view of the fact that many of the students involved in the project had, little previous experience of video production, there were bound to be some obvious 
differences between their initial intentions and the final results. It might be hard to generalize about this. However, the styles of productions and the dramatic themes or concepts the young students selected are clearly connected with the subjective relevance of media models and genres.

2. When interpreting the video films of their fellow students, the production groups involved used very different styles of interpretation. Here again, it is difficult to generalize about this. However, several conclusions can be made. Broadly speaking, a subjective ascription of meaning seems to be connected particularly with the capability (or the possibility) of recognizing personal experiences and forms of knowledge within the text. Here, understanding a video film is not reduced to the cognitive understanding of the content and the suspected intention. There are also emotional-intuitive processes that come into play as soon as symbolic forms are perceived that evoke associations with personal, real life experiences: It seems as if the viewers were only able to assign a subjective meaning to a video whether on a cognitive or an emotional level - if they were able to relate to it in a personal way (Zaslow and Butler, 2002).

3. When asked, some of the young students preferred "open" productions to "closed" ones. That is, if the "message" was too unambiguous (i.e., bold and simple) and did not leave enough space for self-made ascriptions of meaning, these young people often expressed disapproval. Here again, however, there seem to be individual differences in young people's preferences that cannot simply be related to broader social and cultural factors.

4. An observation made during the analysis phase of the gathered data, after presenting the video films to the viewers' sample, showed that girls are more likely to develop an emotional access to video films, whereas boys put more emphasis on formal aspects.

5. The generated results indicated that gender-related differences have more influence on preferences for certain videos and forms of symbolization than differences of age, background, culture, and social-class (Lamb, 2003).

6. The results also pointed out that young viewers showed common reactions to audiovisual symbols used in other students' video productions. This might be interpreted as a confirmation of the assumption that young people make use of an inter-personal understandable, audiovisual symbolic language (Lamb, 2003).

7. Media educators need to support and encourage students' media production activities which help youths to learn about themselves, others, and differential modes of communication. In a media-saturated and deeply complex world as that today, helping youths express themselves and understand others, using all available technologies and strategies, is a goal that is not only desirable but necessary.

8. The support of media lecturers and tutors should not only be concerned with the visual dimension. A subtly differentiated handling of music and sound should be imparted as well. The present study's data analysis suggests that media teachers need a far better knowledge of this area if they are to provide young people with creative alternatives (Featherstone, 1995). Students' knowledge as "consumers" of popular music does not automatically result in an active and productive use of music in their own work. Accurate input, exercises, and suggestions are needed to use music, in conjunction with the visuals, as an independent form of creativity.

9. The selection and presentation of the video productions proved to be a prolonged process. Future similar projects should find ways of publishing these video films in more accessible ways (e.g., via the Internet with message boards for each video where larger samples of viewers can post their comments), and of facilitating dialogues about these productions that are critical as well as appreciative. In this way, they may not be exposed to a selection process of any kind, and this could enhance the communicative dimension of the project's approach, which would definitely be in the students' interest. Moreover, opening up access through the Internet will allow the project to grow more rapidly and attract youths to use technology as a creative tool for dialogue rather than receivers of entertainment.

\section{Ending Note}

One of the advantages a research-based practitioner enjoys is to 'make real' - that is, to rehearse in the field - some of the academic debates that rage in the classroom, in journals and in the conference halls. In the long run, the value of this project and other similar pieces of research work will be measured by two criteria: 'Does this piece of research advance knowledge?' and 'Does this piece of research make an intervention in the field that changes the way others do their work?' For the researcher, living out those debates has been 
one of the richest experiences of the project.

\section{Acknowledgements}

The researcher would like to thank all those students who participated in this research project for their valuable help and assistance.

\section{References}

Bourdieu, P. (1984): Distinction: A Social Critique of the Judgment of Taste. London: Routledge.

Buckingham, D. (1993): Children Talking Television: The Making of Television Literacy. London: Falmer.

Buckingham, D. (1996): Moving Images: Understanding Children's Emotional Responses to Television. Manchester, UK: Manchester University Press.

Buckingham, D. (2000): After the Death of Childhood. Growing Up in the Age of Electronic Media. Cambridge, UK: Polity.

Buckingham, D.; Grahame, J.; and Sefton-Green, J. (1995): Making Media: Practical Production in Media Education. London: English and Media Centre.

Buckingham, D., and Sefton-Green, J. (1994): Cultural Studies Goes to School. London: Taylor and Francis.

Featherstone, M. (1995): Undoing Culture. Globalization, Postmodernism and Identity. London: Sage.

Fisherkeller, J. (2002): Growing Up with Television: Everyday Learning among Young Adolescents. Philadelphia: Temple University Press.

Fisherkeller, J.; Butler, A.; and Zaslow, E. (2000): "What Are You Trying to Say?": New York City Adolescents Interpret Audio-Visual Media Created by Youth in Other Cultures. Report to the International Team of Videoculture. Ludwigsburg, Germany: Institute of Education at Ludwigsburg.

Illich, I. (2001): Tools for Conviviality. 2nd ed. London: Fontana.

Lamb, G. (2003): "VideoCulture in Los Angeles." In: VideoCulture--Video und inter-culture communication. Edited by: Niesyto, H. München, Germany: K- Verlag.

Messaris, P. (1995): Visual Literacy. Boulder, CO: Westview.

Niesyto, H. (2001): "Youth Research on Video Self-Productions in Reflections on a Social-Aesthetic Approach." Visual Sociology. 15:135-53.

Tally, B.; and Kornblum, W. (1997): Student Videos Go to School: How Youth Produced Documentaries Are Used in Six New York City High Schools. A report to the Educational Video Center. New York: Center for Urban Research, City University of New York Graduate Center.

Zaslow, E., and Butler, A. (2002): "That It Was Made by People Our Age Is Better: Exploring the Role of Media Literacy in Transcultural Communication." Journal of Popular Film and Television. 30 (1): 31-40.

\section{Author Information}

Mustafa Mahmoud YOUSRY, PhD, is Associate Professor and researcher at the Department of Photography, Cinema, and Television, Faculty of Applied Arts at Helwan University, Dokki, Giza, Egypt. 